\title{
Interference in Body Area Networks: Are signal-links and interference-links independent?
}

\author{
Andrew (Jian) Zhang*, Leif W. Hanlen, Dino Miniutti*, David Rodda, Ben Gilbert \\ NICTA \\ Canberra, Australia \\ Email: Andrew.Zhang@nicta.com.au
}

\begin{abstract}
Network-to-network interference is a challenging problem in wireless body area networks which move frequently and operate in a non-coordinated way. In this paper, we characterize interference based on field measurements. We examine the correlation and independence between the desired (signal) and interference channels. The results show that over long periods, the two channels are statistically un-correlated. Over a short periods, the channels may become correlated, particularly when the channel variation is small.
\end{abstract}

\section{INTRODUCTION}

Wireless Body-Area-Networks (BANs) represent the next generation in personal area networking [1]. The IEEE-802.15.6 group has provided details of the expectation of such networks. The requirements [2] include an operating range of $3 \mathrm{~m}(6 \mathrm{~m} \times 6 \mathrm{~m} \times 6 \mathrm{~m}$ volume $)$ and up to 10 co-located networks each with up to 256 nodes. The potential for interference from other BANs and also from ISM transmissions - in such networks is large.

One challenging problem is how to mitigate interference between BANs. Typically each BAN (piconet) is associated with one person. When people move - eg. walking in a shopping mall - the density of piconets may become very large: consider the density of people in an elevator, or an entryway. Since these piconets are un-coordinated, one piconet may cause severe interference to another operating network. To investigate the extent of the interference, we conducted measurements in an office environment, using several wearable channel sounder devices, and measured the signal-tointerference ratio (SIR) as a function of time.

In a Body Area Network the signal link will be on-body (ie. between a device on a person and another device on the same person) while the interference link will most likely be off-body (ie. from a device on some other person). Intuition suggests such links ought to be independent over large timescales: movement-induced shadowing is the dominant effect in single user links [8], [9], [10] and movement of participants in a network study is largely independent [11]. However, it is not clear that less frequent events such as stationary subjects, or local congestion (eg. being in an elevator) will be "averaged out" to allow signal-links and interference-links to be analysed independently.

* A. Zhang, L. Hanlen and D. Miniutti also hold research positions with the Faculty of Engineering, Australian National University.

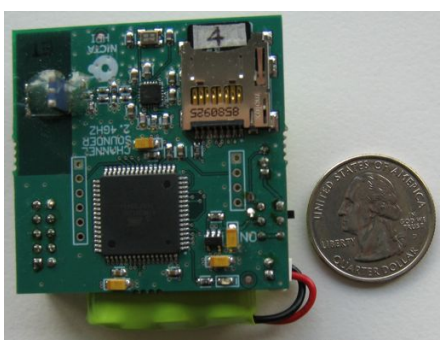

Fig. 1. Wearable channel sounder, at $2400 \mathrm{MHz}$ ISM band. US quarter shown for scale, PCB is approx $50 \mathrm{~mm} \times 40 \mathrm{~mm}$.

We ask: Are the signal and interference links sufficiently independent, such that (eg.) we may measure signal and interference separately, and later combine the results?

This has significant impact for measurement campaigns and simulation work - if the channels are independent, then measurements may be taken without time sycnhronisation and separate measurement campaigns may be combined. In this case, interference on a network may be easily characterized by applying separate on-body and off-body measurements which are widely available [5], [6], [7].

\section{EXPERIMENTAL SETUP}

The interference was measured via a wearable channel sounder, which measures the received signal strength indicator (RSSI) of its operating channel. The sounder is shown in Fig. 1. Each transmitter sends packets with a transmit ID attached. When a channel sounder device receives a packet from another device it $\log$ s the packet ID and RSSI: the ID provides the link identification and the RSSI provides the instantaneous signal strength for that link. Signal-to-Interference (SIR) is found by declaring a given link to be a reference, and other links to be interference. The sensitivity of the transceiver is $-95 \mathrm{dBm}$, and any packet received with a signal strength below this value is dropped. The transceiver was tuned to $2,360 \mathrm{MHz}$ in order to avoid unnecessary cross-talk from local WiFi sources. The experiment used a fixed transmit power of OdBm for all transmitters.

BAN channels are temporally reciprocal (see appendix): down-link and up-link channels at a small time interval are the same. To measure the reference and interference channels, we do not need to separate two received signals simultaneously coming from two transmitters, instead, we can use two 


\begin{tabular}{|c|c|c|c|c|c|}
\hline Person & 1 & 2 & 3 & 4 & 5 \\
\hline \hline Tx & left wrist & left wrist & & & \\
\hline Rx & $\begin{array}{c}\text { left hip } \\
\text { pocket }\end{array}$ & $\begin{array}{c}\text { Right Hip } \\
\text { Pocket }\end{array}$ & $\begin{array}{c}\text { Jacket } \\
\text { Pocket }\end{array}$ & $\begin{array}{c}\text { Jacket } \\
\text { Pocket }\end{array}$ & $\begin{array}{c}\text { Check } \\
\text { Pocket }\end{array}$ \\
\hline
\end{tabular}

TABLE I

SETUP OF THE EXPERIMENT FOR CO-CHANNEL INTERFERENCE MEASUREMENT. EXAMPLE OF ATTACHMENT SHOWN IN FIG. 3(A)

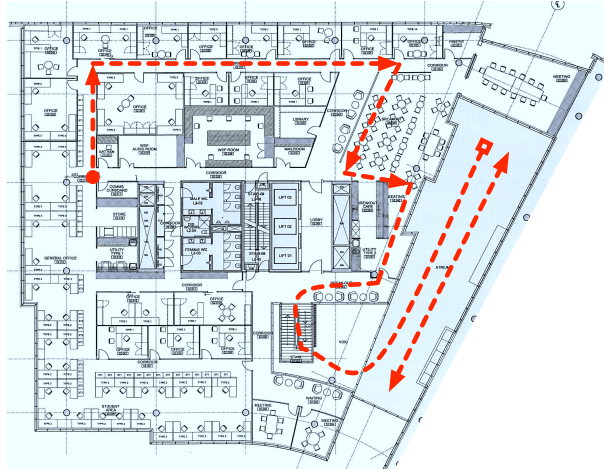

Fig. 2. Floorplan of building NICTA, Canberra, Level 2 and ground-floor where experiment was conducted.

receivers and one transmitter, and apply reciprocity. In the experiment, 2 transmitters (Tx) and 6 receivers (Rx) are used to build a double star network. The setup is shown in Table I. Transmitter-1 sends a packet every 10ms and Tx 2 transmits packets $5 \mathrm{~ms}$ after Tx 1 (so both send packets at the same rate). All receivers $\log$ the RSSI from Tx 1 and 2 to file.

\section{A. Physical arrangement}

The experiment took place over a period of several minutes with subjects walking along the corridor of an office area (see Fig. 2), into a lift area, and up- and down- a large stair well Fig. 3(b). The experiment finished with subjects walking backand forth- in a ground floor atrium, as shown in Fig. 3(c)

In the following analysis, a model of five piconets is established, with each person having a piconet. The on-body and off-body channels are referred as reference and interference channels, respectively. The measurement results are divided into two groups for analysis: In group 1 , user 1 sees cochannel interference from user $2-5$, and in group 2 , user 2 sees interference from users $1,3-5$.

\section{Correlation And IndePEndence Test}

The measured instantaneous signal-to-interference ratio (SIR) is plotted in Fig. 4 for Group 1 and 2, separately. From the figure, we can see that the SIR varies a greatly depending on the link parameters (which subject) and the time of measurement.

The SIR is below OdB on several links for substantial portions of the experiment. The median SIR over the experiment was found to varied between $-5 \mathrm{~dB}$ and $11.5 \mathrm{~dB}$ depending on the link.

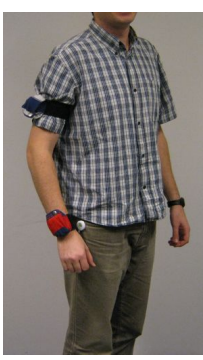

(a) Wearable channel sounder on subject wrist and arm.

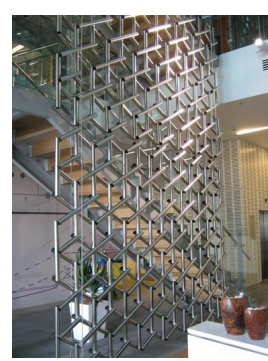

(b) Stairs, aluminium mesh.

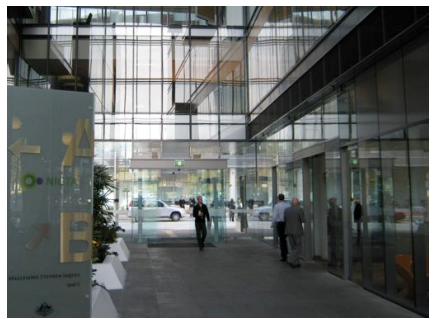

(c) Ground-level corridor, glass enclosed.
Fig. 3. Experimental arrangement, showing typical transceiver placement and various physical environments.
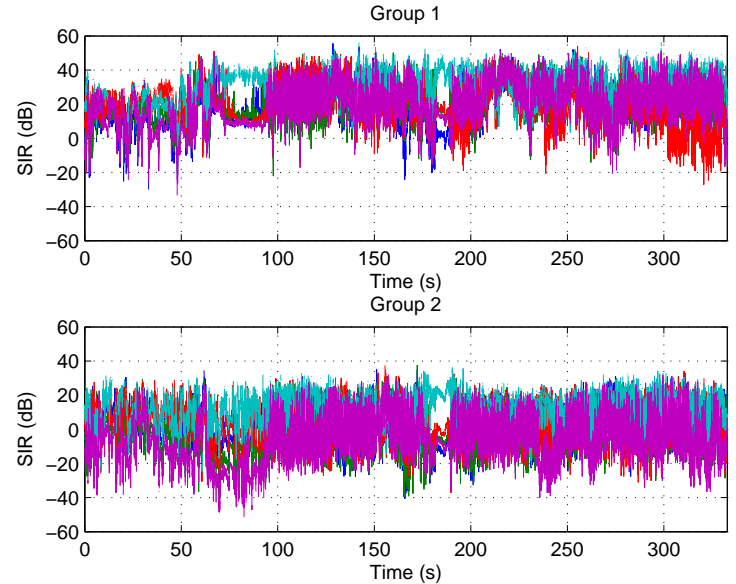

Fig. 4. Instantaneous Signal-to-Interference-Ratio SIR (dB)

We also plot the cumulative density of the SIR for the two extreme links in Fig. ??. The left-most curve gives the worstcase link (for this experiment) and the right-most curve gives the best case.

\section{A. Correlation Test}

The cross-correlation coefficient $\rho(n)$ between the two channels is computed as

$$
\rho\left(m_{0}, n\right)=\frac{\sum_{m=m_{0}}^{m_{0}+M-1} h_{d}(m+n) h_{i}(m)}{\sqrt{\sum_{m=m_{0}}^{m 0+M-1} h_{d}^{2}(m)} \sqrt{\sum_{m=m_{0}}^{m_{0}+M-1} h_{i}^{2}(m)}},
$$

where $h_{d}(m)$ and $h_{i}(m)$ are the pathloss of the reference and interference channels, respectively; $M$ is the span of the 


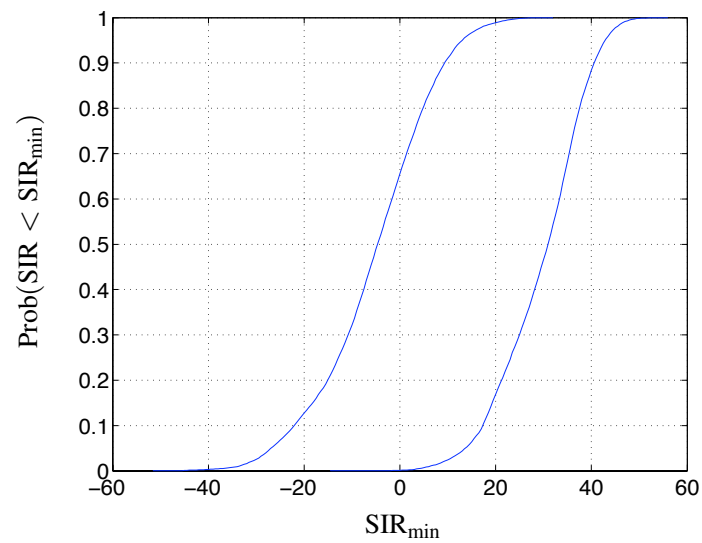

Fig. 5. Cumulative Density for SIR on best (highest SIR) and worst (lowest SIR) link in the experiment. Actual SIR values lie on or between these curves.

correlation window, and $m_{0}$ starts from 1 and increases by 1 each time. The maximum of $\rho\left(m_{0}, n\right)$ for each $m_{0}$ is located at $n=0$ and thus $\rho\left(m_{0}, 0\right)$ is used for presenting the results below.

We first analyze the long-term cross-correlation, with $M=$ 21445 corresponding to a correlation window of $214.45 \mathrm{sec}-$ onds. Fig. 5 shows the probability of correlation coefficient distribution, averaged over all measurements. The figure suggests that the reference and interference channels, over a long observation window, are un-correlated. This matches intuition - if we watch the output of two loosely coupled random processes for long enough, the law-of-large numbers ensures we see low cross-correlation.

We further look at the short-term cross-correlation. In Fig. 6 and 7 , we present the probability of correlation coefficient distribution over a 1 second correlation window for group 1 and 2 measurements, respectively. As can be seen from the two figures, the probability of larger correlation coefficients is significantly increased, for each pair of channels. This suggests that the reference and interference channels are somewhat correlated, over a short observation window. Fig. 8 shows the averaged probability over all measurements.

We have shown [12] that body movement is a significant factor in channel dynamics. It is straightforward to see that both the reference and interference channels will change if the receiver is moving. Higher correlation occurs when the channel is slowly varying which can be seen Fig. 9 and Fig. 10. In particular, around the 75 second point in Fig. 9, one subject held a door open for all other subjects. We interpret this as:

1) When subject movement is the main source of channel dynamics, signal- and interference-links are uncorrelated - since two subjects are not synchronised.

2) When subject movement is not the main source of channel dynamics, signal- and interference-links display common characteristics (and are hence correlated).

Events (item 1), involving subject movement tend to dominate a given "everyday" activity - as seen by the relative frequency of stable-periods of channel path-loss vs unstable periods.

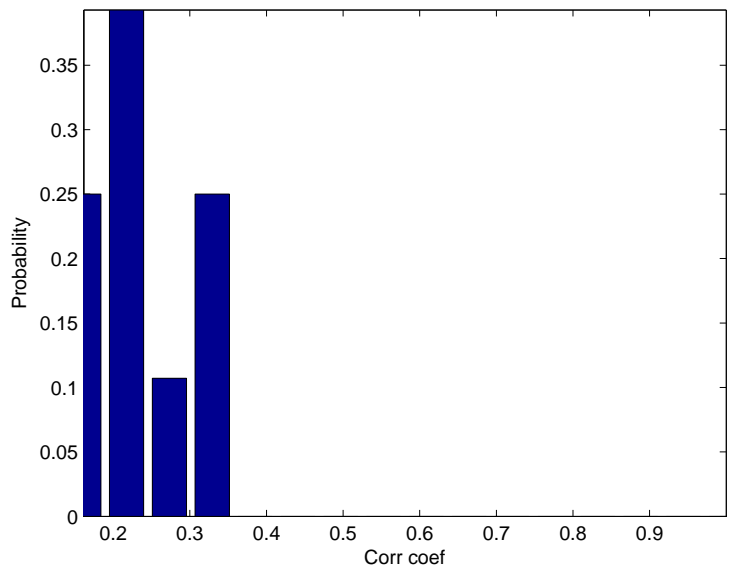

Fig. 6. Probability of correlation coefficient distribution over a 214.45 seconds correlation window

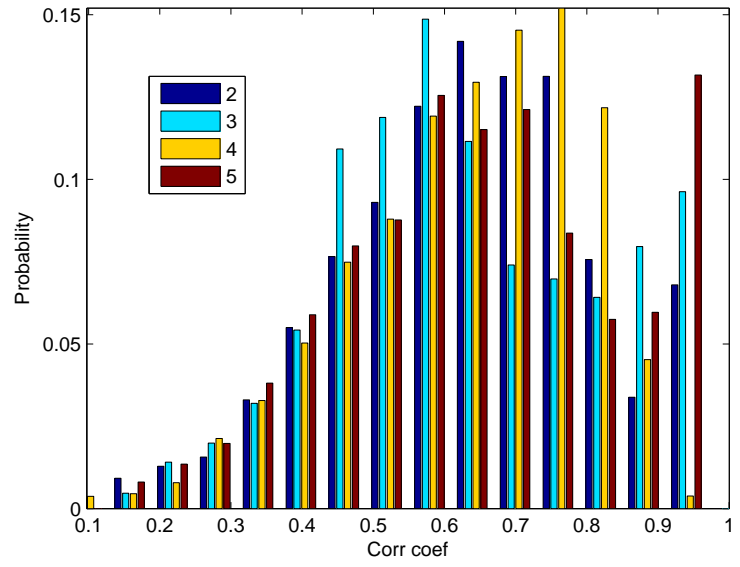

Fig. 7. Probability of correlation coefficient distribution over a 1 second correlation window for group 1 measurement

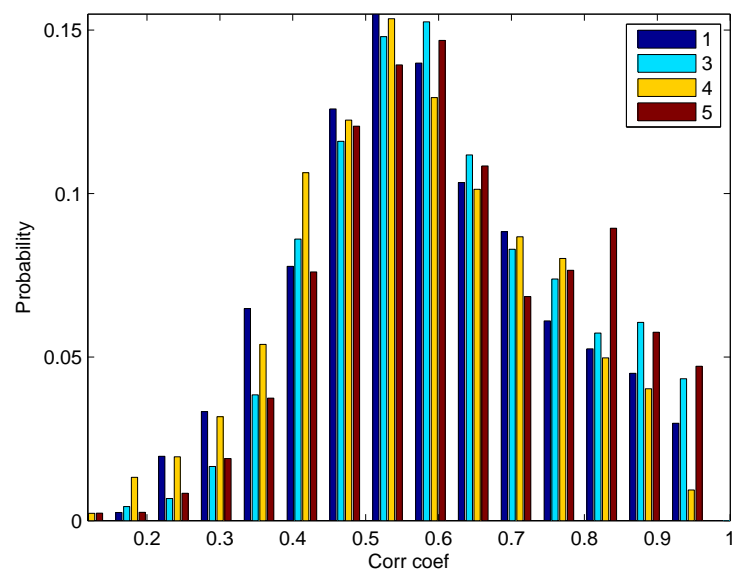

Fig. 8. Probability of correlation coefficient distribution over a 1 second correlation window for group 2 measurement, for all interferers correlated with signal. 


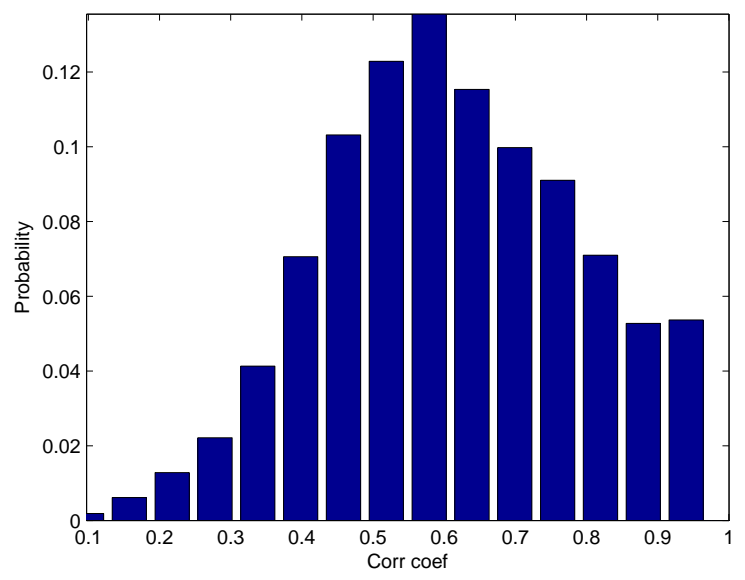

Fig. 9. Probability of correlation coefficient distribution over a 1 second correlation window averaged over all measurements, for all interferers correlated with signal.
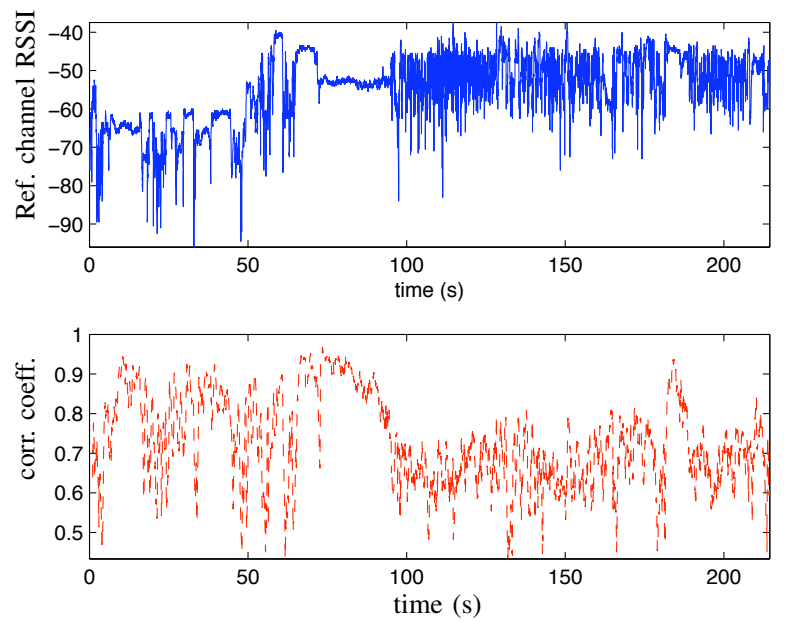

Fig. 10. Relationship between correlation coefficient (with correlation window 1 second) and channel stability. Top: Signal strength over time for reference channel 1; Bottom: Averaged correlation coefficient over time for group 1 measurement.

\section{B. Independence Test}

We further conducted independence test to see whether the reference and interference channels can be regarded as two independent channels. To determine whether two random variables $h_{d}$ and $h_{i}$ are statistically independent, we need to examine whether the following equation holds: $p\left(h_{d}, h_{i}\right)=$ $p\left(h_{d}\right) p\left(h_{i}\right)$. That is, $h_{d}$ and $h_{i}$ are statistically independent if and only if their joint probability mass function (pmf) equals to the product of their respective pmf.

To compute the pmf, the pathloss values in each measurement are quantized separately. 15 quantization bins are used, equally spaced between the maximum and minimum values. The normalized mean squared error (MSE) between $p\left(h_{d}, h_{i}\right)$ and $p\left(h_{d}\right) p\left(h_{i}\right)$ is evaluated for each pair of reference and
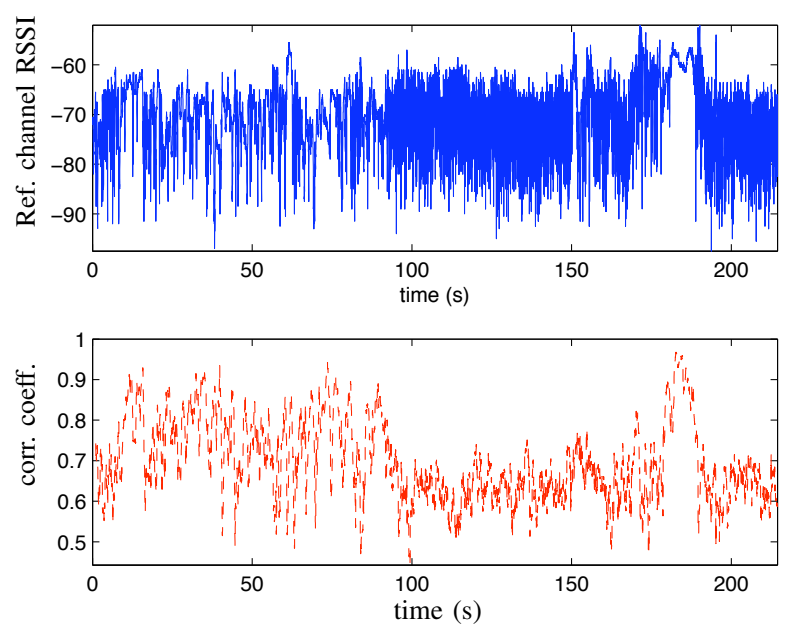

Fig. 11. Relationship between correlation coefficient (with correlation window 1 second) and channel stability. Top: Signal strength over time for reference channel 2; Bottom: Averaged correlation coefficient over time for group 2 measurement.

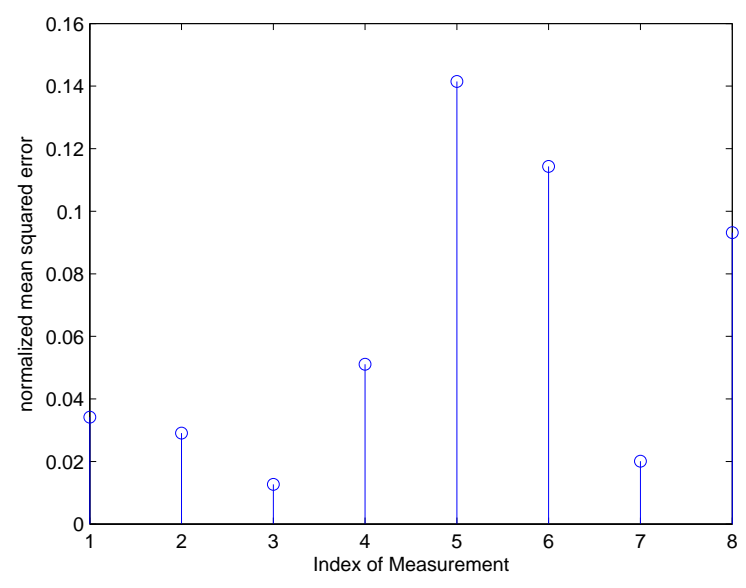

Fig. 12. Normalized MSE of the difference between the joint pmf and the product of 2 pmfs

interference channels. The normalized MSE is defined as

$$
\begin{gathered}
e=\frac{\sum_{k, \ell=1}^{15}\left(p\left(h_{d, k}, h_{i, \ell}\right)-p\left(h_{d, k}\right) p\left(h_{i, \ell}\right)\right)^{2}}{\sum_{k=1}^{15}\left(p\left(h_{d, k}\right)\right)^{2}}, \\
h_{d, k} \in \mathcal{Q}_{k}, h_{i, \ell} \in \mathcal{Q}_{\ell}
\end{gathered}
$$

where $\mathcal{Q}_{k}$ represents the $k$-th quantization bin.

Fig. 11 shows the normalized MSE for 8 pairs of measured channels. These small MSE values suggest that the reference and interference channels are largely independent over a long period.

\section{Conclusions}

Based on field measurements, we have shown that onbody reference- and off-body interference-channels are generally uncorrelated and statistically independent when considered over large periods of time (order 100's of seconds). 


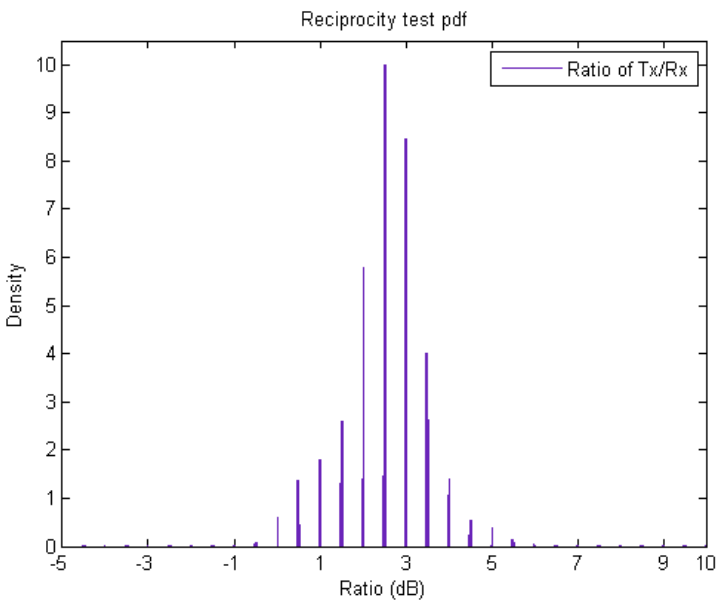

Fig. 13. Distribution of link power difference: uplink $(d B)-\operatorname{downlink}(d B)$. Exact reciprocity occurs when uplink-downlink $=0 \mathrm{~dB}$.

For short time periods, the on-body reference- and off-body interference-channels may show correlation, particularly when the channels vary slowly.

In answering our opening question, we are forced to concede that the correlation between interference and signal links will be highly dependent on the type of activity carried out by the participants. Macroscopic results (eg. median SIR over several minutes) will likely be sensible whether considered from synchronised or un-synchronised data. However, more detailed analysis must make reference to the time-synchronised (or not) nature of the data source.

\section{ACKNOWLEDGEMENT}

NICTA is funded by the Australian Government as represented by the Department of Broadband, Communications and the Digital Economy and the Australian Research Council through the ICT Centre of Excellence program.

\section{APPENDIX}

We briefly outline details of the reciprocity test for the link RSSI. The measurements were taken from a hip-mounted transmitter to a hip-mounted receiver, with a $5 \mathrm{~ms}$ round-trip delay. In Fig. 12 we can see the difference between path loss for up-link and down-link is negligible.

\section{REFERENCES}

[1] A. Astrin, "The promise of body area networks IEEE 802.15.6," Presented, ISMICT 2007, Dec. 2007.

[2] —-, "IEEE 802.15-08-0831-05-0006 TG6 proposal comparison criteria," IEEE submission, Nov. 2008.

[3] D. Davenport, N. Seidl, J. Moss, M. Patel, J. H. A. Batra, S. Hosur, J. Roh, T.Schmidl, O. Omeni, and A. Wong, "MedWiN MAC and security proposal," IEEE802.15.6 technical contribution, document ID: 15-09-0329-00-0006.

[4] D. Miniutti, L. Hanlen, D. Smith, A. Zhang, D. Rodda, B. Gilbert, and G. Fang, "NICTA proposal," IEEE802.15.6 technical contribution, document ID: 15-09-0345-00-0006.

[5] K. Y. Yazdandoost and K. Sayrafian-Pour, "Channel Model for Body Area Network (BAN)," IEEE802.15.6 technical contribution, document ID:15-08-0780-09-0006.
[6] A. Taparugssanagorn, A. Rabbachin, M. Hamalainen, J. Saloranta, and J. Iinatti, "A review of channel modelling for wireless body area network in wireless medical communications," in Proc. 11th Inter. Symp. on Wireless Personal Multimedia Communications (WPMC), 2008.

[7] S. Cotton and W. Scanlon, "An experimental investigation into the influence of user state and environment on fading characteristics in wireless body area networks at $2.45 \mathrm{GHz}$," Wireless Communications, IEEE Transactions on, vol. 8 (1), pp. 6-12, Jan. 2009.

[8] D. B. Smith, L. W. Hanlen, J. A. Zhang, D. Miniutti, D. Rodda, and B. Gilbert, "Characterization of the dynamic narrowband on-body to off-body area channel," in IEEE Intl. Conf. Commun., ICC, Dresden, Germany, June 2009, p. to appear.

[9] J. A. Zhang, D. B. Smith, L. W. Hanlen, D. Miniutti, D. Rodda, and B. Gilbert, "Stability of narrowband dynamic body area channel," IEEE Antennas Wireless Propagat. Lett., p. to appear, 2009.

[10] R. Ganesh and K. Pahlavan, "Effects of traffic and local movements on multipath characteristics of an indoor radio channel," IEE Elect. Lett., vol. 26, pp. 810-812, June 1990.

[11] S. L. Shrestha, A. Lee, J. Lee, and D.-W. Seo, "A group of people acts like a black body in a wireless mesh network," in IEEE Globecom, Washington DC, USA, Nov. 2007, pp. 4834-4839.

[12] J. A. Zhang, D. Smith, L. Hanlen, D. Miniutti, D. Rodda, and B. Gilbert, "Stability of narrowband dynamic body area channel," IEEE Antenna and Wireless Propagation letters, pp. 53-56, 2008. 